\title{
Hydrodynamic and Chemical Factors in Clogging by Montmorillonite in Porous Media
}

\author{
David C. Mays ${ }^{\star}$ and \\ Department of Civil Engineering, University of Colorado at Denver and Health Sciences Center
}

James R. Hunt

Department of Civil and Environmental Engineering, University of California at Berkeley

\begin{abstract}
Clogging by colloid deposits is important in water treatment filters, groundwater aquifers, and petroleum reservoirs. The complexity of colloid deposition and deposit morphology preclude models based on first principles, so this study extends an empirical approach to quantify clogging using a simple, one-parameter model. Experiments were conducted with destabilized suspensions of sodiumand calcium-montmorillonite to quantify the hydrodynamic and chemical factors important in clogging. Greater clogging is observed at slower fluid velocity, consistent with previous investigations. However, calcium-montmorillonite causes one order of magnitude less clogging per mass of deposited particles compared to sodium-montmorillonite or a previously published summary of clogging in model granular media. Steady state conditions, in which the permeability and the quantity of deposited material are both constant, were not observed, even though the experimental conditions were optimized for that purpose. These results indicate that hydrodynamic aspects of clogging by these natural materials are consistent with those of simplified model systems, and they demonstrate significant chemical effects on clogging for fully destabilized montmorillonite clay.
\end{abstract}

\section{Introduction}

Permeability reduction caused by deposition of colloidal particles in porous media, or clogging, is important in numerous environmental processes, including water treatment $\left(e . g .{ }^{1}\right)$, aquifer storage and recovery $\left(e . g .{ }^{2}\right)$, surface water-ground water interaction $\left(e . g .{ }^{3}\right)$, formation damage in petroleum reservoirs $\left(\right.$ e.g. ${ }^{4}$, chapter 8$)$ and the influence of earthquakes on hydrology $\left(e . g .{ }^{5}\right)$. Filtration theory $\left(6^{-8}\right)$ assists in understanding particle clogging, because it predicts the porosity reduction that results from particle accumulation. However, filtration theory is not sufficient to predict clogging because there is no unique relationship between porosity and permeability (9). The thrust of this study is to investigate the factors that control the degree of clogging for a given quantity of deposited particles. This focus makes the present study distinct from, but complimentary to, parallel approaches that predict permeability from the geometry of the porous medium in the absence of particles (e.g. 10$)$, and from network models that predict permeability from an assumed porosity-permeability relationship in elements with particle accumulation (e.g., 11$)$.

Clogging is known to depend on physical factors, which control hydrodynamic effects, and on chemical factors, which control the colloidal stability of the particles (12). In order to investigate hydrodynamic effects on clogging, Mays and Hunt (13) analyzed published data sets from constant-flow filtration experiments that measured particle accumulation and head

* Corresponding author: david.mays@ cudenver.edu, phone (303) 352-3933, fax (303) 556-2368. 
loss over a range of fluid velocities. Data were analyzed using a one-parameter clogging model based on the work of O'Melia and Ali (14), who predicted clogging based on the surface area within the porous media, which increases with particle deposition:

$$
\frac{\Delta H}{\Delta H_{o}}=(1+\gamma \sigma)^{2}
$$

where $\Delta \mathrm{H}$ is the head loss, $\Delta \mathrm{H}_{\mathrm{O}}$ is the clean bed head loss, $\sigma$ is the specific deposit, defined as the dimensionless ratio of deposit volume to total filter volume, and $\gamma$ is an empirical clogging parameter that quantifies clogging per specific deposit. Because of the difficulty in measuring specific area (15), this model requires an empirical approach. The fact that $\gamma$ is not constant reflects the important role of deposit morphology. One physical interpretation of $\gamma$ is that its inverse is a critical specific deposit such that for $\sigma>1 / \gamma$, deposited particles contribute most of the surface area within the porous medium, resulting in greater clogging per unit of additional particle accumulation.

The analysis of published clogging data by Mays and Hunt (13) revealed a power-law correlation between the empirical clogging parameter and the Peclet number of the flow, $\gamma=$ $10^{6} N \mathrm{Pe}^{-0.55 \pm 0.09}$, consistent across the various data sets, that indicated greater clogging at smaller Peclet numbers, where the Peclet number is defined as

$$
N_{P_{e}}=\frac{u d_{c}}{D_{p}},
$$

and $D_{p}$ is the particle diffusivity given by

$$
D_{p}=\frac{\kappa T}{3 \pi \mu d_{p}},
$$

where $\kappa$ is Boltzmann's constant, $T$ is temperature, $\mu$ is dynamic viscosity, and $d_{p}$ is the primary particle diameter. Three of the data sets analyzed by Mays and Hunt (13) used kaolinite clay, but with a relatively small range of velocity. The most extensive data set analyzed was Veerapaneni's (16, chapter 5), who used a velocity range of almost two orders of magnitude, but these results were for latex microspheres depositing on glass beads. Considering these limitations, the first goal of the present study was to investigate whether the power-law correlation between clogging and hydrodynamic loading reported by Mays and Hunt (13) also extends to clogging by natural materials conducted over a wide range of velocity.

With regard to chemical effects, cation valence is known to affect the permeability of soils containing clays (e.g., 17-18) and to influence the morphology of clay aggregates (e.g.' 19-21). However, experiments using soils or cores make it difficult to quantify the relationship between chemical conditions, permeability, and deposit morphology for clays because of two confounding factors: First, with soil or core samples, one cannot quantify particle accumulation by mass balance on the influent and effluent suspensions, which prevents the analysis of clogging for a given specific deposit. This is important, because knowing the specific deposit is required to determine the role of deposit morphology. Second, most studies on soil permeability focus on the dramatic effects that result when chemical changes, illustrated on Quirk-Schofield diagrams (22), create newly stabilized or newly destabilized particles. This focus on chemical changes makes it difficult to isolate the role of cation valence for a given degree of particle stabilization. Accordingly, the second goal of this study is to isolate the effect of cation valence on clogging during filtration experiments using a fully destabilized natural clay mineral. 
A third goal of this study is to investigate long-term clogging. The conventional application of filtration theory to granular filters, which are frequently backwashed, provided little incentive to consider long-term effects. In contrast, these effects are important in natural environments where backwashing is not possible. Particle transport and clogging may be conceptualized as a dynamic feedback process between fluid flow and particle deposition: Flow transports particles, which form deposits, which cause clogging, which reduces or redirects subsequent fluid flow. Although classic filtration studies simultaneously modeled particle transport and clogging $(14,23)$, contemporary studies on environmental particle transport seldom address clogging. However, an appreciation for the feedback process between fluid flow and particle deposition is demonstrated by reports showing that fluid shear (24) or hydrodynamic drag (25) can mobilize previously deposited particles $(26,27)$. As long-term deposition proceeds under constant flow conditions, one might expect this dynamic feedback process to approach a limit, or steady state condition, in which the average deposition rate matches the average mobilization rate, with constant permeability and specific deposit. Steady state conditions could then be used as a baseline from which to evaluate permeability changes resulting from hydraulic, mechanical, or chemical perturbations. In the filtration literature, several reports document either permeability (28) or specific deposit (29) approaching a constant value, but simultaneous observations of steady state for both variables have not been reported. The third goal of the present study, therefore, was to investigate the potential for steady state clogging in long-term experiments.

\section{Materials and Methods}

The apparatus was designed to promote rapid achievement of steady state, with large approach velocities and concentrated influent suspensions to maximize particle delivery, destabilized particles to maximize particle accumulation, a short column to minimize the volume in which deposition and mobilization must reach equilibrium, and durations of tens of thousands of pore volumes. The filter column had a diameter of $1.4 \mathrm{~cm}$, a length of $2.0 \mathrm{~cm}$ and was packed with Clementine silica (Prime Equipment, South San Francisco, CA), with density $2.65 \mathrm{~g} / \mathrm{cm}^{3}$, passing a \#80 $(177 \mu \mathrm{m})$ mesh and retained on a \#100 $(150 \mu \mathrm{m})$ mesh, resulting in a geometric mean diameter of $163 \mu \mathrm{m}$. Sieved sand was rinsed with and then stored in distilled water. The column was wet-packed in short lifts and a carbon dioxide flood was used to remove trapped air bubbles. The porosity was 0.35 and the pore volume was $1.09 \mathrm{~cm}^{3}$. The sand was kept in place by a stainless steel mesh at the top and bottom of the column. Pressure ports were installed at the top, bottom and an intermediate location $0.7 \mathrm{~cm}$ from the top. No samples were collected from the intermediate pressure ports, since such an effort would have disturbed the deposits (23). The head loss across the entire filter and across the top section was measured with Validyne DP15 pressure transducers (Validyne Engineering, Northridge, CA).

Montmorillonite was selected as a model particle because it is a common clay mineral that is present in soils from temperate regions, sedimentary rocks, and poorly drained environments (30), whose morphology depends on cation valence ( $20 ; 31$, chapter 10$)$, allowing investigation of chemical effects on clogging. Montmorillonite SWy-2 was purchased from the Source Clays Repository (Clay Minerals Society, West Lafayette, IN). Clay was mixed with deionized water and then centrifuged at $100 \mathrm{G}$ to remove particles with equivalent diameter greater than $2 \mu \mathrm{m}$, assuming Stokes settling, clay density of $2.33 \mathrm{~g} / \mathrm{cm}^{3}$, and a settling path of $7 \mathrm{~cm}$ perpendicular to the centrifuge axis. This method of size fractionation has been shown to be superior to membrane filtration for colloidal suspensions (32). A series of ion exchange rinses were used to convert the raw clay into Na-montmorillonite or Ca-montmorillonite (33). Suspensions of $100 \mathrm{mg} / \mathrm{L} \mathrm{Na-montmorillonite} \mathrm{or} \mathrm{Ca-montmorillonite,} \mathrm{with} \mathrm{pH}$ in the range of 6.0-6.2, were stirred rapidly and continuously to minimize aggregation, then pumped by a peristaltic pump at constant flow through the column. Destabilized conditions were imposed by using a background solution of $100 \mathrm{mM} \mathrm{NaCl}$ or $2 \mathrm{mM} \mathrm{CaCl}_{2}$, which are approximately one order of 
magnitude above the critical coagulation concentrations of $8 \pm 6 \mathrm{mM}$ for Na-montmorillonite or $0.12 \pm 0.02 \mathrm{mM}$ for Ca-montmorillonite (31, chapter 10). The column was flushed with at least 15 pore volumes of particle-free brine prior to each experiment, which determined the clean bed head loss $\Delta \mathrm{H}_{\mathrm{o}}$. Ca-montmorillonite suspensions included $3 \mathrm{~g} / \mathrm{L}$ of 2 -chloroacetamide biocide, added as a precaution to extend the shelf life of effluent samples before turbidity measurements. This concentration of 2-chloroacetamide did not change the $\mathrm{pH}$ nor electrical conductivity of a $2 \mathrm{mM} \mathrm{CaCl}_{2}$ solution, and caused no significant increase in turbidity, indicating that the biocide had no influence on particle stability.

Effluent samples were collected by an automatic $25 \mathrm{~mL}$ fraction collector. The turbidity of these samples and the influent suspension was measured with a Hach $2100 \mathrm{~A}$ turbidimeter (Hach Company, Ames, IA) with a nominal error of $\pm 0.07 \mathrm{NTU}$ for $100 \mathrm{mg} / \mathrm{L} \mathrm{Na}$ -

montmorillonite and $\pm 0.7 \mathrm{NTU}$ for $100 \mathrm{mg} / \mathrm{L} \mathrm{Ca}$-montmorillonite, converted to concentrations using clay-specific calibration curves, and then used to calculate the specific deposit, $\sigma$, as a function of time by mass balance.

Mass recovery was determined by comparing the measured specific deposit determined by extrusion to the calculated specific deposit from an overall mass balance. The extruded sample was vigorously shaken to ensure complete clay mobilization into a particle-free brine matching that used in the relevant experiment $\left(100 \mathrm{mM} \mathrm{NaCl}\right.$ or $\left.2 \mathrm{mM} \mathrm{CaCl}_{2}\right)$. The contribution from suspended particles in the pore space was negligible. Preliminary experiments were conducted to confirm that flow through the filter was one-dimensional, that there was no deposition in the supply tubing, that particle mobilization from the silica sand was negligible, and that the magnitude of peristaltic pulsing was within the $\pm 2.2 \mathrm{~cm}$ nominal error of the pressure transducers. Further details are available the Supporting Information and in (34, chapter 4). Experimental conditions are listed in Table 1.

\section{Results and Discussion}

Raw data for experiment 1 are shown in Figure 1, with corresponding results for other experiments shown in Figures S5-S12 of the Supporting Information. The first few concentration measurements indicate that breakthrough occurred rapidly, so the influent concentration was only slightly higher than the effluent during the remainder of the experiment. This observation was typical and reflects the objective of rapidly achieving steady state. The head loss, shown in the lower panel, increased with time in both the top and bottom sections of the filter. Mass balance results are discussed in the Supporting Information.

\section{Montmorillonite Clogging in Silica Sand}

Clogging data were analyzed by plotting the normalized increase in head loss, $\Delta H / \Delta H_{o^{-}}$, versus the specific deposit, $\sigma$, for the first 3500 pore volumes of each experiment, both averaged over the whole filter. This duration provides a uniform basis to compare results across experiments, and eliminates the effect of head loss through the cake deposit that formed in experiments operated beyond 5000 pore volumes, which is modeled differently (35). Results for Na-montmorillonite with $u=0.021,0.11$, and $0.53 \mathrm{~cm} / \mathrm{s}$ are shown in Figures 2-4, respectively. To illustrate the profound effect of cation valence, Figure 2 also shows results for Ca-montmorillonite at $u=0.021 \mathrm{~cm} / \mathrm{s}$. The Na-montmorillonite results in Figure 2 correspond to the data of Figure 1. For clarity in this and similar figures, data were selected to display up to 25 points, but all concentration data and their corresponding head loss data were used for model fitting by least squares. Error bars were estimated using the method described in the Supporting Information. Figure 2 also shows the fitted clogging model, which on average differs from the data by a factor of $e^{R M S E}$, where $R M S E$ is the root mean squared error, defined in the Supporting Information. For experiment $1, R M S E=0.52$, so on average the model differs from the data by a factor of $e^{0.52}=1.7$. During the first 3500 pore volumes, the critical specific 
deposit $\sigma=1 / \gamma$ was exceeded only for experiments $1,2 \mathrm{~A}$, and 4 , shown as dotted vertical lines on Figures 2 and 3. Fitted parameters for all experiments are given in the "whole filter" column of Table 1, and results for Ca-montmorillonite at $\mathrm{u}=0.21$ and $0.53 \mathrm{~cm} / \mathrm{s}$, which maintained essentially clean bed conditions, are shown in Figures S13-S14 of the Supporting Information.

Although the fitted models do not replicate the observations in detail, they do provide an average description that can be used to parameterize hydrodynamic and chemical effects. For a given specific deposit, the data clearly show major differences in clogging. The model serves to quantify those differences, because its single parameter is uniquely defined by the given data. The fitted values of $\gamma$ are plotted against Peclet number, $N_{\mathrm{Pe}}$, in Figure 5 along with a power-law correlation for Na-montmorillonite and for Ca-montmorillonite, assuming primary particle diameter $d_{p}=1 \mu \mathrm{m}$, consistent with the clay preparation procedure. The uncertainty of the power-law exponent is a 95\% confidence interval calculated using the standard error for the slope of the linear regression of $\ln (\gamma)$ versus $\ln \left(N_{\mathrm{Pe}}\right)$. The resulting exponents are significantly different from 0 and -1 , but not significantly different from each other.

The first goal of this study was to determine whether clogging by natural materials is consistent with the empirical correlation reported in Mays and Hunt (13), shown on Figure 5. Both Naand Ca-montmorillonite have power law exponents that are comparable to previously reported results. This analysis indicates that the hydrodynamic effect on clogging is also applicable to natural materials, which supports the apparently general nature of this scaling relationship for porous media clogging by destabilized, abiotic particles at constant flow. This empirical relationship is consistent with simulations predicting that at higher flow rates, the resulting ballistic deposits will be more streamlined, and therefore require more colloids in order to exert the same drag on the fluid (36).

\section{Cation Valence}

The second goal of this study was to isolate the effect of cation valence on clogging with a fully destabilized clay. As shown in Figure 5, the Peclet number scaling is consistent across data sets, but the magnitude of the clogging parameter depends strongly on cation valence. Assuming that the biocide did not affect the Ca-montmorillonite experiments, which is reasonable given that it was non-ionic and had no significant impact on turbidity, Figure 5 shows that Na-montmorillonite causes approximately 20 times more clogging per mass of deposited clay than Ca-montmorillonite. This difference is consistent with the known swelling behavior of Na-montmorillonite (30) that would cause increased clogging per mass of clay, and with the formation of multi-plate quasi-crystals by Ca-montmorillonite (20) that would cause decreased clogging per mass of clay. The formation of quasi-crystals results from the outer-sphere complexation of $\mathrm{Ca}^{2+}$ between two opposing siloxane cavities on the montmorillonite surface, resulting in a characteristic plate spacing of approximately $2 \mathrm{~nm}$ (31, chapter 10).

A speculative interpretation of this 20 -fold difference in clogging is that quasi-crystal formation by Ca-montmorillonite increases the effective particle size from the perspective of clogging. Specifically, if characteristic particle size for Ca-montmorillonite were 100 times larger than Na-montmorillonite, then the calculated Peclet number would shift to the right by two orders of magnitude, yielding a more consistent correlation for $\gamma$.

\section{Steady State}

To investigate the potential for steady state clogging, the raw head loss data were analyzed for the full duration of each experiment. In order to eliminate clogging by cake deposits, only data from the lower $1.3 \mathrm{~cm}$ of the filter were considered, which prevented inclusion of experiment $2 \mathrm{~B}$, for which no intermediate head loss data were available. The final slope was determined 
by linear regression of head loss versus pore volumes for the final $20 \%$ of each experiment, with a 95\% confidence interval determined from the standard error of the estimated slope. The $20 \%$ interval was arbitrary, but was chosen to focus on the final slope while avoiding erroneous results caused by noise in final few head loss data. The results, given in the "lower filter" column of Table 1, indicate that only experiments 1,2A, and 4 had significant particle accumulation that exceeded the critical specific deposit of $\sigma=1 / \gamma$. These experiments were associated with low fluid velocity $(u \leq 0.11 \mathrm{~cm} / \mathrm{s})$ and large final head loss $\left(\Delta \mathrm{H} / \Delta \mathrm{H}_{\mathrm{O}}>5\right)$ that was still increasing upon termination (slope $>1 \mathrm{~cm} / 10,000 / \Delta \mathrm{H}_{\mathrm{o}}$ ), indicating a lack of steady state. Minimal deviation from clean bed conditions was observed in the remaining experiments: high fluid velocity $(u \geq 0.21 \mathrm{~cm} / \mathrm{s})$ appears to have prevented significant deposit accumulation $\left(\sigma<1 / \gamma\right.$ during the first 3500 pore volumes), resulting in small final head loss $\left(\Delta \mathrm{H} / \Delta \mathrm{H}_{\mathrm{O}}<2\right)$ with minimal head loss increase at termination (slope $<1 \mathrm{~cm} / 10,000 / \Delta \mathrm{H}_{\mathrm{o}}$ ).

These experiments suggest that no steady state clogging should be expected for fluid velocities characteristic of slow sand filtration $(u \approx 0.01 \mathrm{~cm} / \mathrm{s})$, groundwater flow $(u \approx 0.001 \mathrm{~cm} / \mathrm{s})$, or soil infiltration $(u \approx 0.0001 \mathrm{~cm} / \mathrm{s})$. This negative result does not prove steady state clogging is impossible, but it was surprising given that the experiment was specifically designed for this goal (small filter, concentrated influent suspension, destabilized particles, and up to 61,000 pore volumes of influent). Deposition in natural environments is subjected to variations in suspension concentration, composition, aqueous chemistry, and hydrodynamic loading that would likely preclude steady state clogging in nature.

\section{Hydrodynamic Shear}

Head loss can be interpreted in terms of hydrodynamic shear, either as a shear rate or a shear stress with a mean shear rate, $G$, in porous media (37) given by:

$$
\mathrm{G}=\left(\frac{\rho \mathrm{gu} \Delta \mathrm{H}}{\varepsilon \mu \mathrm{L}}\right)^{1 / 2},
$$

where $\rho$ is fluid density, $g$ is gravity, $\Delta \mathrm{H}$ is head loss in the lower $\mathrm{L}=1.3 \mathrm{~cm}$ of the filter, and porosity is $\varepsilon=0.35$. The mean shear stress, $\tau=\mu \mathrm{G}$, provides insight into the strength of the particle deposits. The results, shown in Table 2 , indicate significant clogging occurs when clean bed shear rates, $G_{0}$, are less than or equal to $700 \mathrm{~s}^{-1}$, and negligible clogging occurs for $\mathrm{G}_{\mathrm{o}} \geq$ $1400 \mathrm{~s}^{-1}$. This suggests a critical shear rate between $700 \mathrm{~s}^{-1}$ and $1400 \mathrm{~s}^{-1}$ or shear stress between 7 and 14 dyne $/ \mathrm{cm}^{2}$ that will prevent significant accumulation of destabilized montmorillonite. Although this hydrodynamic shear appears to have prevented deposit initiation, it was not sufficient to remove existing deposits, as evidenced by the final shear stress of 16 dyne $/ \mathrm{cm}^{2}$ in experiment $2 \mathrm{~A}$, which did not reach steady state. The apparent deposit shear strengths in experiments $1,2 \mathrm{~A}$, and 4 between 4 and $16 \mathrm{dyne} / \mathrm{cm}^{2}$ are similar to those for $\mathrm{Na}-$ montmorillonite depositing in fractures, $\tau=1-8 \mathrm{dyne} / \mathrm{cm}^{2}$ (33), and to kaolinite depositing in porous media, $\tau=2.7-4$ dyne $/ \mathrm{cm}^{2}$ (38). The continued increase in head loss, hydrodynamic shear, and deposit strength reflects the dynamic feedback between fluid flow and particle deposition leading to dynamic deposits that evolve over time.

The results of this study indicate that the specific deposit is not sufficient to predict clogging by montmorillonite, since the data clearly show hydrodynamic and chemical effects. The orderof-magnitude differences in clogging for a given specific deposit indicate an important role for deposit morphology. The empirical parameter $\gamma$ can be used to quantify the important role of macroscopic hydrodynamic and chemical effects on clogging (Figure 5), but continued progress toward a mechanistic understanding of deposit morphology, extending the work of Veerapaneni and Wiesner (36), will require experimental techniques that non-invasively 
quantify deposit structure $($ e.g. 39,40$)$. This effort, in turn, would provide the basis for improved pore-scale clogging models.

\section{Supplementary Material}

Refer to Web version on PubMed Central for supplementary material.

\section{Acknowledgements}

The authors thank Boris Faybishenko and the Earth Sciences Division at Lawrence Berkeley National Laboratory for providing the laboratory facility, and the four anonymous reviewers whose insightful comments improved the manuscript. Financial support was provided by an Office of Civilian Radioactive Waste Management Fellowship (to DCM), administered by Oak Ridge Institute for Science and Education under a contract between the U.S. Department of Energy and Oak Ridge Associated Universities, and by the National Institute of Environmental Health Sciences Superfund Basic Research Program, grant 3PH2-ES04705.

\section{References}

1. Yao KM, Habibian MT, O'Melia CR. Water and waste water filtration: Concepts and applications. Environ Sci Technol 1971;5(11):1105-1112.

2. ASCE. Standard Guidelines for Artificial Recharge of Ground Water, EWRI/ASCE 34-01. American Society of Civil Engineers; Reston, VA: 2001.

3. Packman AI, MacKay JS. Interplay of stream-surface exchange, clay particle deposition, and streambed evolution. Water Resour Res 2003;39(4):1097.10.1029/2002wr001432

4. Tiab, D.; Donaldson, E. Petrophysics. Gulf Publishing Company; Houston: 1996.

5. Montgomery DR, Manga M. Streamflow and water well responses to earthquakes. Science 2003;300:2047-2049. [PubMed: 12829774]

6. Ryan JN, Elimelech M. Colloid mobilization and transport in groundwater. Colloids Surf A 1996;107:1-56.

7. Bradford SA, Simunek J, Bettahar M, van Genuchten MT, Yates SR. Modeling colloid attachment, straining, and exclusion in saturated porous media. Environ Sci Technol 2003;37(10):2242-2250. [PubMed: 12785531]

8. Johnson WP, Li X, Tong M. Colloid retention behavior in environmental porous media challenges existing theory. Eos Trans Am Geophys Union 2005;86(18):179-180.

9. Babadagli T, Al-Salmi S. A review of permeability-prediction methods for carbonate reservoirs using well-log data. Soc Petrol Eng Reservoir Eval Eng 2004;7(2):75-88.

10. Zhao F, Landis HR, Skerlos SJ. Modeling of porous filter permeability via image-based stochastic reconstruction of spatial porosity calculations. Environ Sci Technol 2005;39(1):239-247. [PubMed: 15667100]

11. Shapiro AA, Bedrikovetsky PG, Santos A, Medvedev OO. A stochastic model for filtration of particulate suspensions with incomplete pore plugging. Transp Porous Media. 200610.1007/ s11242-006-0029-5

12. Compere F, Porel G, Delay F. Transport and retention of clay particles in saturated porous media: Influence of ionic strength and pore velocity. J Contam Hydrol 2001;49:1-21. [PubMed: 11351511]

13. Mays DC, Hunt JR. Hydrodynamic aspects of particle clogging in porous media. Environ Sci Technol 2005;39(2):577-584. [PubMed: 15707058]

14. O'Melia CR, Ali W. The role of retained particles in deep bed filtration. Prog Water Res 1978;10(56): $167-182$.

15. Emmanuel S, Berkowitz B. Mixing-induced precipitation and porosity evolution in porous media. Adv Water Resour 2005;28:337-344.

16. Veerapaneni, S. Ph.D. thesis. Rice University; Houston, TX: 1996. Formation and morphology of colloidal deposits in porous media.

17. Shainberg I, Letey J. Response of soils to sodic and saline conditions. Hilgardia 1984;52(2):1-57. 
18. Suarez DL. Sodic soil reclamation: Modelling and field study. Australian J Soil Res 2001;39(6):12251246.

19. Chen JS, Cushman JH, Low PF. Rheological behavior or Na-montmorillonite suspensions at low electrolyte concentration. Clays Clay Min 1990;38(1):57-62.

20. Mohan KK, Fogler HS. Effect of $\mathrm{pH}$ and layer charge on formation damage in porous media containing swelling clays. Langmuir 1997;13:2863-2872.

21. Heller H, Keren R. Rheology of Na-rich montmorillonite suspension as affected by electrolyte concentration and shear rate. Clays Clay Min 2001;49(4):286-291.

22. Lebron I, Suarez DL. Variations in soil stability within and among soil types. Soil Sci Soc Am J 1992;56:1412-1421.

23. Ives KJ, Pienvichitr V. Kinetics of the filtration of dilute suspensions. Chem Eng Sci 1965;20:965973.

24. Bergendahl J, Grasso D. Prediction of colloid detachment in model porous media: Hydrodynamics. Chem Eng Sci 2000;55:1523-1532.

25. Li X, Zhang P, Lin CL, Johnson WP. Role of hydrodynamic drag on microsphere deposition and reentrainment in porous media under unfavorable conditions. Environ Sci Technol 2005;39(11):40124020. [PubMed: 15984777]

26. Bai R, Tien C. Transient behavior of particle deposition in granular media under various surface interactions. Colloids Surf A 2000;165:95-114.

27. Kim J, Tobiason JE. Particles in filter effluent: The roles of deposition and detachment. Environ Sci Technol 2004;38(22):6132-6138. [PubMed: 15573617]

28. Adin A, Rebhun MA. model to predict concentration and head-loss profiles in filtration. J Am Water Works Assoc 1977;69:444-453.

29. Hunt JR, Hwang BC, McDowell-Boyer LM. Solids accumulation during deep bed filtration. Environ Sci Technol 1993;27(6):1099-1107.

30. Borchardt, G. Smectites, Chapter 14. In: Dixon, JB.; Weed, SB., editors. Minerals in Soil Environments. 2nd. Soil Science Society of America; Madison, WI: 1989.

31. Sposito, G. The Chemistry of Soils. Oxford University Press; New York: 1989.

32. Gimbert LJ, Haygarth PM, Beckett R, Worsfold PJ. Comparison of centrifugation and filtration techniques for the size fractionation of colloidal material in soil suspensions using sedimentation field-flow fractionation. Environ Sci Technol 2005;39(6):1731-1735. [PubMed: 15819231]

33. Kessler, JH. Ph.D. Thesis. University of California; Berkeley, CA: 1993. Transport and channeling effects in a fracture partially clogged with colloidal material.

34. Mays, DC. Ph.D. thesis. University of California; Berkeley, CA: 2005. Hydrodynamics of particle clogging in saturated granular media: Analysis and experiments.

35. Lee DJ, Wang CH. Theories of cake filtration and consolidation and implications to sludge dewatering. Water Res 2000;34(1):1-20.

36. Veerapaneni S, Wiesner MR. Deposit morphology and head loss development in porous media. Environ Sci Technol 1997;31(10):2738-2744.

37. Ives KJ. Rapid filtration. Water Res 1970;4:201-223.

38. Ives KJ, Fitzpatrick CSB. Detachment of deposits from sand grains. Colloids Surf A 1989;39(13): 239-253.

39. Leis AP, Schlicher S, Franke H, Strathmann M. Optically transparent porous medium for nondestructive studies of microbial biofilm architecture and transport dynamics. Appl Environ Microbiol 2005;71(8):4801-4808. [PubMed: 16085878]

40. Li X, Lin CL, Miller JD, Johnson WP. Pore-scale observation of microsphere deposition at grain-tograin contacts over assemblage-scale porous media domains using x-ray microtomography. Environ Sci Technol 2006;40(12):3762-3768. [PubMed: 16830539] 

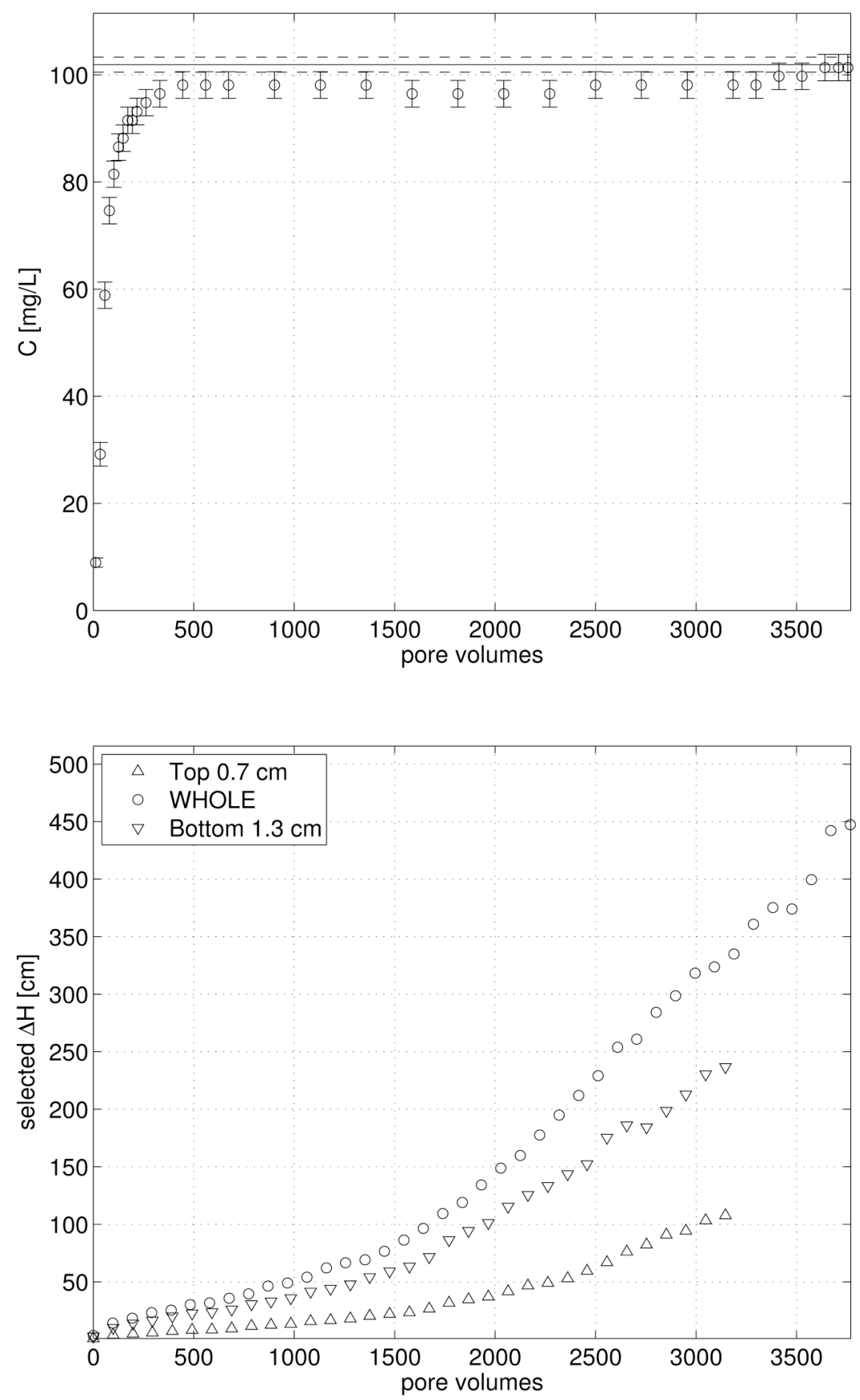

Figure 1.

Effluent concentration (top) and head loss (bottom) versus pore volumes eluted, experiment 1. The influent concentration, $C_{o}$, is shown as a solid line with an error of one standard deviation shown by dashed lines. The error bars on the head loss data, which were selected to display only 40 measurement times, are smaller than the plotted symbols. Head loss measurements in the top section were discontinued when the head loss exceeded the transducer capacity at approximately $100 \mathrm{~cm}$. 


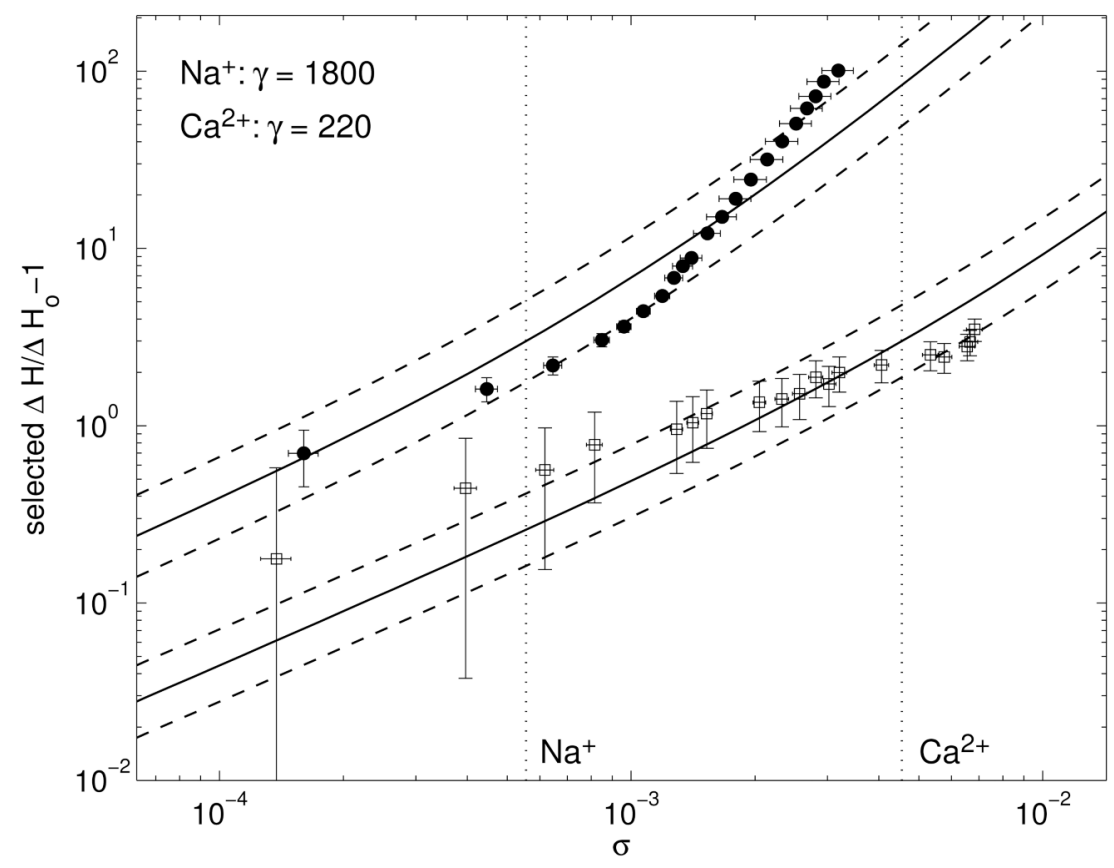

Figure 2.

Normalized increase in head loss versus specific deposit for experiments with $\mathrm{u}=0.021 \mathrm{~cm} /$ s. Experiment $1\left(\mathrm{Na}^{+}\right)$is shown with solid circles. Experiment $4\left(\mathrm{Ca}^{2+}\right)$ is shown with open squares. For each, the solid line is the fitted clogging model, and the dashed lines are plus or minus one root mean squared error (RMSE). The critical specific deposit occurs at $5.6 \times 10^{-4}$ with $\mathrm{Na}^{+}$and at $4.5 \times 10^{-3}$ with $\mathrm{Ca}^{2+}$. 


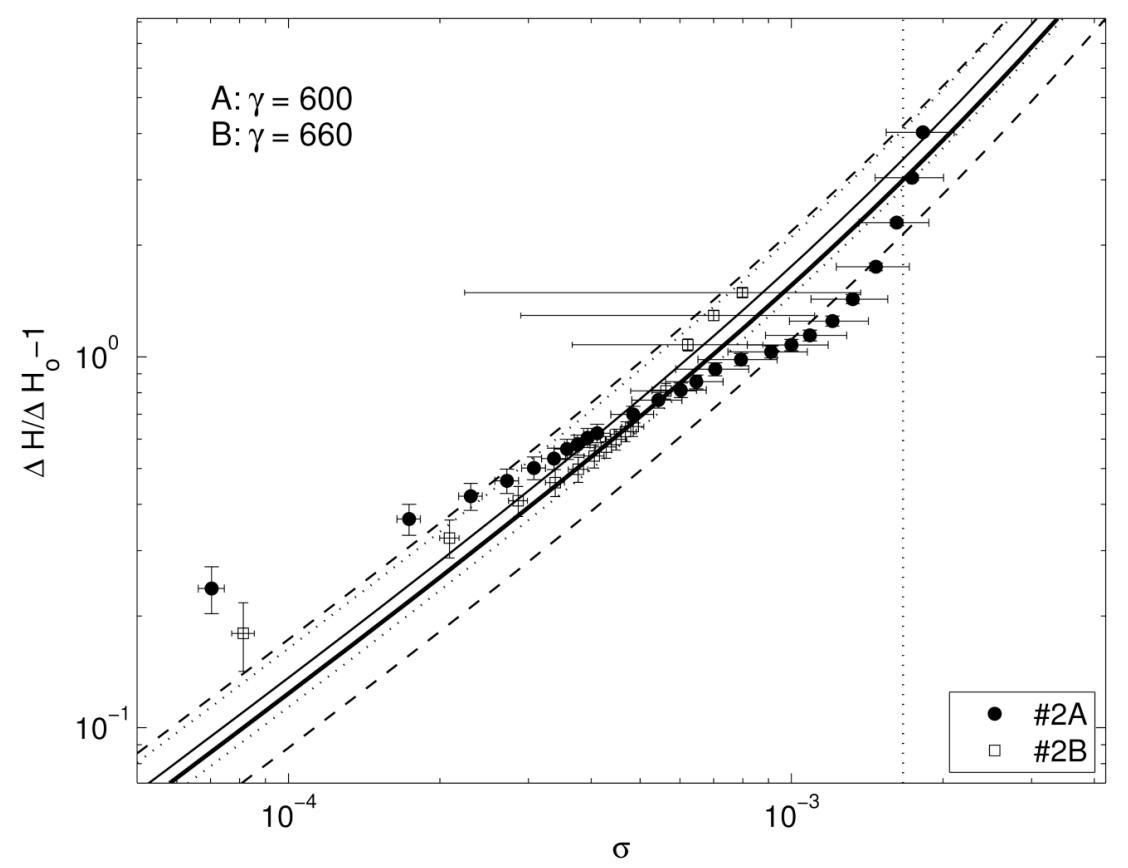

Figure 3.

Normalized increase in head loss versus specific deposit for experiment 2 . The bold line is the fitted model and the dashed lines are \pm RMSE for replicate A; the regular line is the fitted model and the dotted lines are \pm RMSE for replicate $B$. Rapid clogging in experiment $2 \mathrm{~A}$ is expected above the dotted vertical line at $\sigma=1 / \gamma$. 


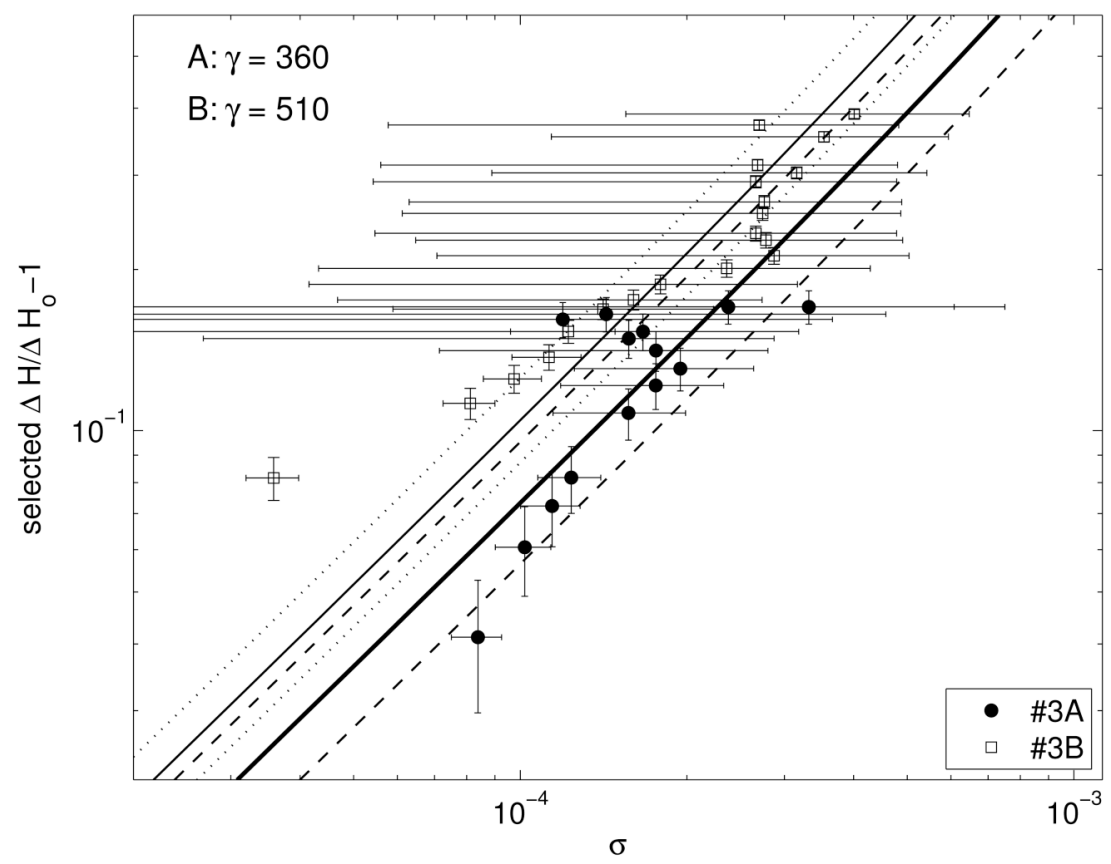

Figure 4.

Normalized increase in head loss versus specific deposit for experiment 3 . The bold line is the fitted model and the dashed lines are \pm RMSE for replicate A; the regular line is the fitted model and the dotted lines are \pm RMSE for replicate $B$. 


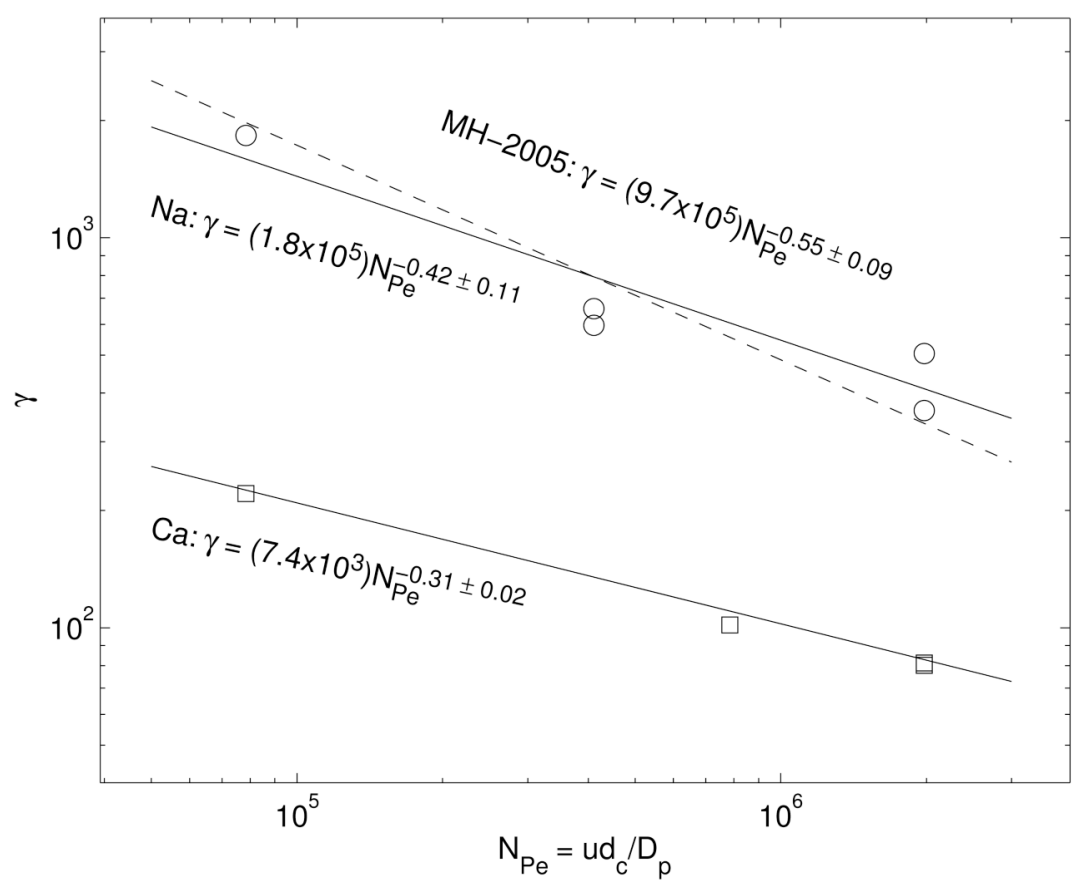

Figure 5.

Fitted clogging parameter $\gamma$ versus Peclet number for Na-montmorillonite (circles) and Camontmorillonite (squares). Note there are two squares at $N_{\mathrm{Pe}}=2 \times 10^{6}$. The dashed line is the empirical correlation reported by Mays and Hunt (13), which summarized the effect of fluid velocity on clogging in six previously published data sets. 


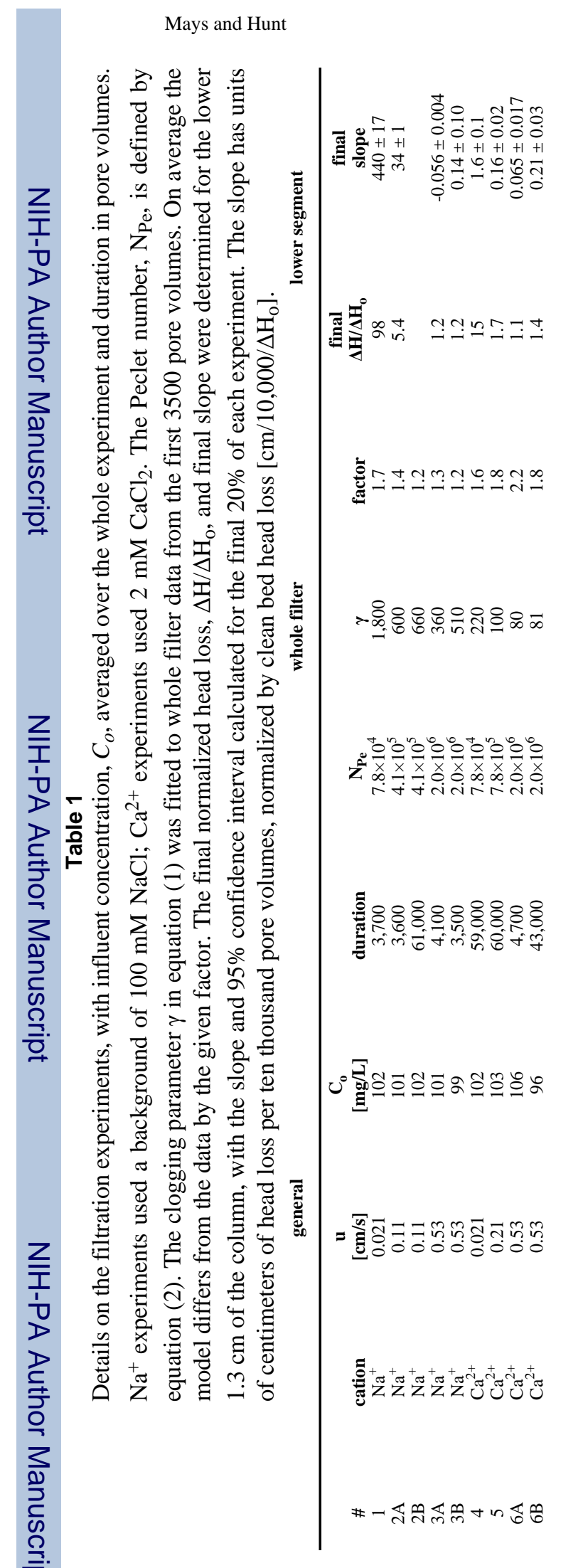

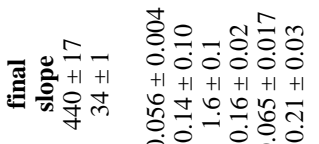

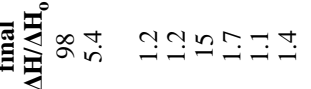

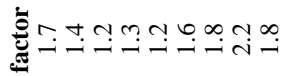

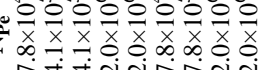

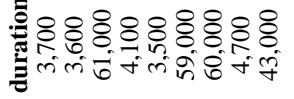

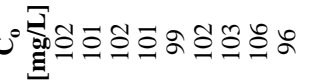

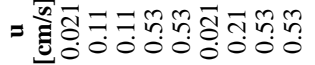

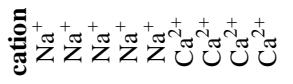

Environ Sci Technol. Author manuscript; available in PMC 2008 September 16. 


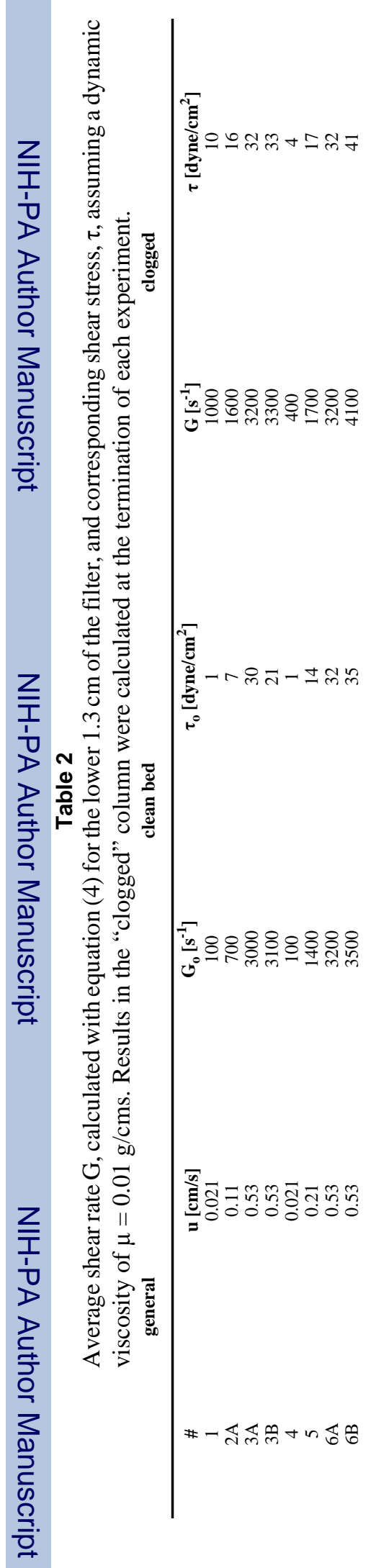

